\title{
Componentes de la asociatividad empresarial y gobernanza en Áreas Naturales Protegidas con capacidades turísticas
}

\section{Components of business associativity and governance in Protected Natural Areas with tourist capacities}

\author{
Dulce María Lucero López* \\ Alberto Francisco Torres García*
}

\begin{abstract}
This article analyzes the concepts of business associativity and governance with emphasis in Protected Natural Areas (PA) dedicated to the provision of tourist services. Thus, the definition of business associativity and its possible contributions to economic development are presented. At the same time, the concept of governance is examined as element in the implementation of associative models. The methodology used is exploratory. The results obtained are the determination of the components that constitute the associative processes, as well as a conceptual framework that explains the importance of governance in the fulfilment of community objectives.
\end{abstract}

Keywords: tourism, Protected Natural Areas, business associativity, governance.

\begin{abstract}
Resumen
El presente artículo tiene como propósito analizar los conceptos de asociatividad empresarial y gobernanza, con énfasis en Áreas Naturales Protegidas (ANP) dedicadas a la prestación de servicios turísticos. Para ello, se define el concepto de asociatividad empresarial y sus posibles contribuciones al desarrollo económico. Igualmente, se examina la noción de gobernanza como elemento en la implementación de modelos asociativos. La metodología empleada es de tipo exploratoria. Los resultados obtenidos son la determinación de los componentes que integran los procesos de asociatividad, así como un marco conceptual que explica la importancia de la gobernanza en el cumplimiento de los objetivos comunitarios.
\end{abstract}

Palabras clave: turismo, Áreas Naturales Protegidas, asociatividad empresarial, gobernanza.

* Universidad Autónoma de Baja California Sur, correos-e: dulce.lucerolopez@gmail.com y atorresg@uabcs.mx 


\section{Introducción}

La expansión de turismo se ha manifestado en la generación de beneficios como la modernización de los medios de transporte, el aumento del empleo y la generación de ingresos a partir de la prestación de servicios turísticos; incluso en localidades que, aunque sean pequeńas o remotas, cuentan con atractivos para cubrir la demanda turística. En estas áreas, el turismo es la principal fuente de ingresos debido a las condiciones desventajosas que enfrentan en comparación con las áreas urbanas en términos de centralización y recursos; por lo que pequeñas empresas deben formar alianzas y organizaciones entre ellas con el objetivo común de ser más competitivas (Galvez-Acevedo et al., 2020). Precisamente la Organización Mundial del Turismo (OMT), en el Código Ético Mundial para el Turismo (OMT, 1999), señala que las poblaciones y comunidades locales se asociarán a las actividades turísticas y tendrán una participación equitativa en los beneficios económicos, sociales y culturales que reporten, especialmente en la creación directa e indirecta de empleo.

En el caso de México, es preciso mencionar que entre los activos más valorados se tiene la diversidad cultural y natural, la cual contribuye a la atracción de una considerable demanda, incluyendo lugares donde las actividades económicas propias de las regiones pueden ser limitadas; tal es el caso de los territorios decretados patrimonio de la humanidad y ANP (Ibañez, 2014). Así, considerar las actividades turísticas como medios para el fomento del desarrollo económico de las pequeñas localidades o comunidades situadas en ANP es base para el correcto aprovechamiento de los recursos de manera responsable. En ese sentido, el desarrollo desempeña un papel determinante para la potencialización de la economía, donde éste sería un proceso en el que participan actores clave para la discusión de asuntos del mismo territorio (Gallicchio, 2004). No obstante, el desarrollo turístico es un arma de doble filo para las comunidades locales y su actitud afecta directamente el desarrollo actual y futuro (Hanafiah et al., 2013).

El turismo puede ser un agente de cambio de gran alcance, pero con demasiada frecuencia la planificación de la industria se basa únicamente en criterios económicos aislados (Murphy, 1983). Por ende, las empresas desempeñan un rol significativo. En el ámbito empresarial, la competencia subyace en la capacidad de participar en una industria que favorezca el surgimiento de nuevas formas de ventajas competitivas, las cuales, en algunos casos, pueden generarse a través de asociaciones. Así, impulsar acciones en conjunto y fomentar la cultura de la asociatividad entre los empresarios emerge como una iniciativa para la generación de ventajas competitivas y el desarrollo económico de regiones o localidades. 
La asociatividad surge como un mecanismo de cooperación que persigue la creación de valor a través de la solución de problemas comunes que se originan por falta de escala (Liendo y Martínez, 2001). Al respecto, en cuanto a la influencia que presenta la asociatividad como medio para el desarrollo de ventajas competitivas, ésta se constituye como una herramienta que ayuda a impulsar el desarrollo local, pues al aumentar la competitividad se estimulan las iniciativas que ayudan a diversificar las operaciones empresariales (Fundeanu y Sandu, 2014). Al asociarse, el tejido empresarial de un determinado territorio puede fortalecerse y consolidar su participación en el mercado, incrementar su poder de negociación y atraer financiamiento, disminuir los costos en las adquisiciones y compras de materiales, articular procesos de capacitación en temas de interés común, mejorar su cadena de valor y, en general, favorecer las capacidades de producción. Para ello, es necesario que los procesos asociativos se realicen con un alto nivel de compromiso, voluntad, sin egoísmos y con mucha confianza entre los empresarios, pensando siempre en el interés del grupo en común y en el desarrollo propio de las empresas (Arguello et al., 2017).

Así, la asociatividad es considerada como un modelo viable entre los procesos de desarrollo local, tanto por fomentar la mejora de la calidad de vida de las personas como por satisfacer las necesidades humanas que van en crecimiento (Varisco, 2008). De tal forma que en un mundo globalizado, adoptar estrategias de asociatividad empresarial en micro, pequeńas y medianas empresas es clave para el desarrollo y fortalecimiento de éstas (Bada et al., 2017). Particularmente, en relación con los destinos turísticos, se busca que los modelos de asociatividad lleven a los actores a operar de manera conjunta y lograr beneficios para la comunidad (Barba y Molina, 2008).

Derivado de las conceptualizaciones anteriores, se puede inferir que la asociatividad presenta una oportunidad de desarrollo para los territorios que deciden implementarla, tomando en cuenta siempre las condicionantes mínimas para su correcta ejecución. Con relación a estas condiciones, Tañski et al. (2011) han determinado algunas categorías que facilitan la asociatividad y la eventual conformación de algún modelo como clústeres, redes empresariales o alianzas estratégicas. Una de estas categorías fue denominada como relacionamiento, integrándose por las variables de gobernanza y capital social. Respecto a las variables, Tañski et al. señalan que:

la presencia de la categoría 'gobernanza' resultó necesaria, ya que organizarse dentro de un sector con miras a elevar las condiciones de competitividad desde la cooperación imponía haber logrado: determinado grado de autonomía, estructura de 
poder empresarial consolidado, confianza generalizada, liderazgo empresarial reconocido por los pares, democratización de las determinaciones que se pudieran consensuar entre los miembros del sector, y otros factores no menores; todo esto exigía abordar el estudio de la gobernanza (2011: 104).

Es conveniente recalcar que la gobernanza funge como un factor determinante al momento de implementar prácticas que tienen incidencia en el desarrollo económico y social de las comunidades. En lo que a este trabajo se refiere, las experiencias que se presentan en relación con el turismo alternativo (ecoturismo, turismo rural y turismo comunitario) suelen ser heterogéneas, debido a que su grado de consolidación ha dependido de la capacidad de estructurar y fortalecer sus instituciones comunitarias y sistemas de gobernanza, pues resultan elementos claves en la regulación del manejo de bienes comunes vinculados a esta actividad, al igual que el desarrollo de emprendimientos con mejor desempeño en su gestión y estabilidad a lo largo del tiempo (Palomino et al., 2016).

En vista de lo anterior, el presente trabajo tiene como objetivo analizar el concepto de asociatividad empresarial y los principales componentes que inciden en la ejecución de modelos asociativos, así como la relación de éstos con la generación del desarrollo y ventajas competitivas a nivel territorial. El análisis mencionado se genera desde la perspectiva de los servicios turísticos en ANP, con el fin de propiciar una mejora en la oferta de los servicios a través de su integración. Con ello, se pretende resaltar las características y los requerimientos mínimos que deben presentar los territorios para poder activar modelos asociativos en entornos de tipo turístico.

\section{Metodología}

Para su realización, el enfoque metodológico que se desarrolla en este artículo es de tipo exploratorio, a través de la búsqueda y revisión de artículos indexados en bases de datos de reconocimiento científico como Scopus, Elsevier, Redalyc, DOAJ, Latindex, Science Direct, Scielo y Jstor. Se seleccionaron artículos descriptivos relacionados con asociatividad, gobernanza y servicios turísticos, teniendo una selección entre el periodo 20142019, para efectuar un análisis más completo y actualizado. Igualmente se incluyen aportes de varios años atrás respecto al periodo señalado, esto como una referencia de la evolución que han tenido los conceptos y su aplicación. Los textos seleccionados se analizaron de manera crítica para lograr cumplir el objetivo de este artículo, tomando en cuenta los casos de estudio desarrollados en comunidades que, en su mayoría, presentan 
las mismas características en cuanto al desarrollo del territorio y las actividades económicas realizadas.

Para la integración de este artículo se presenta el desarrollo de la literatura de manera estructurada y con el apoyo de tablas para sintetizar la información, se contrastaron algunas conceptualizaciones generadas y se analizaron de manera individual los componentes que las integran. Los resultados se componen de un modelo conceptual sobre la asociatividad en territorios declarados ANP, cuya característica principal versa sobre la prestación de servicios turísticos; la figura de dicho modelo fue elaborada mediante el software ATLAS.ti (2017), en su versión 8. Por último, se establecen las conclusiones, compuestas por los principales hallazgos y las deficiencias encontradas en la línea de investigación abordada.

\section{Conceptualización de asociatividad y su importancia en el desarrollo de los territorios y ventajas competitivas}

Una parte importante de la competitividad de las empresas se genera al exterior de las mismas, en las relaciones que éstas logran establecer con su entorno (Dini, 2010). Para Vázquez-Barquero (2005), las relaciones de las empresas en un entorno determinado afectan el comportamiento de la productividad y los procesos de crecimiento, al igual que los cambios estructurales de las mismas; por ende, las redes empresariales desempeñan un papel notable en el desarrollo de los territorios, pues éstas crean las condiciones para hacer eficiente la organización de la producción y la mejora de los sistemas productivos de las regiones que las ponen en práctica. El mismo autor señala que estas colaboraciones se pueden dar de diversas formas como redes personales de empresarios, agrupaciones, alianzas estratégicas o clústeres.

Ante la necesidad de alternativas de desarrollo entre integrantes de una comunidad o territorio, la unificación de éstos se hace cada vez más necesaria y posibilita compartir responsabilidades y potencialidades, especialmente a nivel local, con el fin de plantear estrategias donde la suma genere un esfuerzo y un tejido de interacciones compartidas, capaces de dinamizar un futuro más prometedor implementando proyectos innovadores y particulares (Posada et al., 2017). Así, la asociatividad forma parte de una de las estrategias más reconocidas con relación al desarrollo local, siendo ésta un mecanismo mediante el cual diferentes organizaciones unen esfuerzos, voluntades, iniciativas y recursos, para el logro de objetivos en común (Rosales, 1997 en Muñoz, 2019).

En la tabla 1 se muestran los conceptos más relevantes que se han generado a lo largo de estos ańos; esto con el fin de poder contrastar y 
realizar un análisis más detallado de cada uno de los componentes que lo conforman.

Tabla 1

Matriz de conceptos de asociatividad empresarial

\begin{tabular}{|c|c|}
\hline Autor & Concepto \\
\hline Rosales (1997) & $\begin{array}{l}\text { Mecanismo de cooperación entre empresas pequeñas y medianas, } \\
\text { donde cada empresa que participa mantiene su independencia } \\
\text { jurídica y su autonomía gerencial, decidiendo voluntariamente } \\
\text { participar en un esfuerzo en conjunto con otros participantes para } \\
\text { lograr un objetivo en común. }\end{array}$ \\
\hline $\begin{array}{l}\text { Poliak, 2001, en } \\
\text { Liendo y } \\
\text { Martínez (2001) }\end{array}$ & $\begin{array}{l}\text { Estrategia de colaboración colectiva que está vinculada a negocios } \\
\text { concretos. Las empresas, en ese contexto, desarrollan un esfuerzo } \\
\text { colectivo para la concreción de objetivos comunes, que pueden ser } \\
\text { muy disímiles. }\end{array}$ \\
\hline $\begin{array}{l}\text { Secretaría de } \\
\text { Turismo (Sectur, } \\
\text { 2001) }\end{array}$ & $\begin{array}{l}\text { Concentración de empresas en una misma región que realiza acti- } \\
\text { vidades económicas similares, donde las relaciones fomentan el } \\
\text { desarrollo de múltiples interdependencias de ellas ofreciendo forta- } \\
\text { lecimiento en relación con la competitividad. }\end{array}$ \\
\hline $\begin{array}{l}\text { Liendo y } \\
\text { Martínez (2001) }\end{array}$ & $\begin{array}{l}\text { Mecanismo de cooperación que persigue la creación de valor a } \\
\text { través de la solución de problemas comunes que surgen por falta de } \\
\text { escala. }\end{array}$ \\
\hline $\begin{array}{l}\text { Dini et al. } \\
(2007)\end{array}$ & $\begin{array}{l}\text { Capacidad que tienen las empresas de permanecer en el mercado } \\
\text { donde no depende exclusivamente de los recursos de las mismas, } \\
\text { sino también de modalidades de comunicación, relacionamiento e } \\
\text { intercambio que éstas establecen con otras empresas o instituciones } \\
\text { del sistema productivo en que actúan. }\end{array}$ \\
\hline $\begin{array}{l}\text { Cogollo y } \\
\text { Arrieta (2014) }\end{array}$ & $\begin{array}{l}\text { Mecanismo que se refleja en el desarrollo local y se convierte en } \\
\text { agente de desarrollo, donde se está completamente convencido de } \\
\text { la necesidad que se tiene de trabajar de manera conjunta para poder } \\
\text { superar situaciones de tipo pobreza, exclusión y desigualdad social. }\end{array}$ \\
\hline $\begin{array}{l}\text { Fajardo et al. } \\
\text { (2016) }\end{array}$ & $\begin{array}{l}\text { Estrategias aplicables para la optimización de recursos, aumento en } \\
\text { los niveles de ventas y fomento de las interrelaciones comerciales; } \\
\text { esto a través de la formación de grupos de afinidad, gremios y sobre } \\
\text { todo la implementación de redes de cooperación entre negocios o } \\
\text { empresas con algún tipo de afinidad. }\end{array}$ \\
\hline $\begin{array}{l}\text { Guerrero y } \\
\text { Villamar (2016) }\end{array}$ & $\begin{array}{l}\text { Determina los factores que intervienen en los procesos de asociati- } \\
\text { vidad comunitaria, donde éstos llevarán a una mejora en la asocia- } \\
\text { tividad en el marco del desarrollo comunitario, considerando } \\
\text { factores económicos (competitividad, desarrollo económico indivi- } \\
\text { dual y colectivo) y no económicos (capital social y cultura). }\end{array}$ \\
\hline
\end{tabular}


Tabla 1 (continuación)

\begin{tabular}{|l|l|}
\hline \multicolumn{1}{|c|}{ Autor } & \multicolumn{1}{|c|}{ Concepto } \\
\hline Estrada (2016) & $\begin{array}{l}\text { Determinación de un territorio para la implementación de un } \\
\text { modelo de desarrollo, la unión de los integrantes de la comunidad } \\
\text { para el logro de objetivos en común y la participación de actores } \\
\text { externos. }\end{array}$ \\
\hline $\begin{array}{l}\text { Melo } \text { et al. } \\
(2017)\end{array}$ & $\begin{array}{l}\text { Estrategias que ofrecen mejora del nivel de vida de la población, } \\
\text { organización de la comunidad, mejora de la productividad, forta- } \\
\text { lecimiento de la competitividad, conservación y uso sostenible del } \\
\text { territorio. }\end{array}$ \\
\hline $\begin{array}{l}\text { Posada } \text { et al. } \\
(2017)\end{array}$ & $\begin{array}{l}\text { Estrategias implementadas para llevar a cabo la acción de compartir } \\
\text { responsabilidades y potencialidades a nivel local, presentada en una } \\
\text { asociatividad de tipo funcional: ejecución de acciones para el cum- } \\
\text { plimiento de un objetivo central; unión de los intereses comunes, } \\
\text { capacidad de articulación. }\end{array}$ \\
\hline
\end{tabular}

Fuente: elaboración propia con base en (Posada et al., 2017), (Melo et al., 2017), (Estrada, 2016), (Guerrero y Villamar, 2016), (Fajardo et al., 2016), (Cogollo y Arrieta, 2014), (Dini et al., 2007), (Liendo y Martínez, 2001), (Sectur, 2001), Poliak 2001, en Liendo y Martínez (2001), (Rosales, 1997).

El compromiso que se genera al momento de implementar modelos asociativos es muy importante, porque puede derivar en la generación de empleos y otros beneficios a través de la vinculación de los miembros de la comunidad. Asimismo, la asociatividad permite el establecimiento de convenios directos e indirectos con instituciones y organizaciones (Cogollo y Arrieta, 2014), para ser más competitivo en el mercado. Como señala North (1993 en Sánchez, 2010), las pautas institucionales facilitan la configuración de relaciones estables de confianza y cooperación en la producción de bienes públicos y en la conformación de actores sociales comprometidos con el orden jurídicamente fundado. Así, la estrategia de asociatividad puede funcionar como unión de los intereses comunes; lo natural como la base y lo social es lo que establece la ruptura o la unión; por ello, el ejercicio de la asociatividad se refleja en la capacidad de articulación (Posada et al., 2017).

Igualmente, la asociatividad ayuda a mejorar el nivel de vida de la población que la práctica, a través de la organización de la comunidad, la productividad, la reducción de costos y una participación más estable en el mercado, mediante el aprovechamiento de las economías a escala, la vinculación y la cooperación creando, así, capital social y confianza. A decir de Gutiérrez y Silva (2013), la asociatividad surge como una alternativa para que un conjunto de entidades territoriales desarrolle procesos de cooperación persiguiendo crear valor. 
Guerrero y Villamar (2016) afirman que las empresas que buscan asociarse tienen la posibilidad de ingresar a nuevos mercados o consolidarse en los existentes, diversificar la demanda, exportar aprovechando el cambio estacional, obtener menores costos de producción y acceder a nuevos conocimientos. Asimismo, Estrada afirma que:

La asociatividad y la acción colectiva se enmarcan en procesos de desarrollo que parten del territorio, con actores que tejen fuertes lazos de confianza y reciprocidad, con un fuerte componente de articulación entre actores territoriales y extraterritoriales y la creación de instituciones formales e informales para la autoorganización y el logro de los acuerdos comunes. Todo lo anterior viabiliza la gobernanza territorial, gracias a la articulación entre el Estado y las comunidades, en torno a las diferentes dimensiones del desarrollo como la política, la económica, la ambiental, la social y la cultural (2016: 148).

No obstante, si bien el desarrollo local permite generar cercanía entre los integrantes de las comunidades y les adjudica un nivel de poder (Gallicchio, 2004), es importante considerar cuestiones propias de ciertos modelos que fomentan el desarrollo en las comunidades a través de la asociatividad, debido a que algunos han presentado fallas en su diseño y ejecución. Dicha situación hace necesario analizar el concepto de gobernanza y su papel en el funcionamiento de modelos de asociatividad.

\section{Gobernanza como elemento principal en la implementación de modelos de asociatividad}

El estudio de la gobernanza se presenta como un campo que trata de incluir la idea de que lo político y lo institucional son básicos para estructurar la cuestión pública y alcanzar objetivos de múltiples alcances. Para lograr la gobernanza existen algunos elementos imprescindibles como el equilibrio entre Estado y sociedad, la cooperación entre actores, la coherencia en las acciones emprendidas, eficiencia y efectividad en la acción pública, horizontalidad en el relacionamiento e información y la rendición de cuentas. De igual forma, la gobernanza se puede considerar como un factor de innovación para encontrar soluciones creativas a problemas complejos, basándose en una dinámica de trabajo entre personas con un alto manejo de confianza, compromiso y negociación (Arroyo y Palafox, 2017).

Para Simancas y Ledesma (2017) la gobernanza implica a múltiples actores que tienen interés en una tarea específica. Estos actores forman redes de interacciones continuas, causadas por la necesidad de intercambiar recursos y negociar objetivos compartidos. La identificación de los actores es fundamental para garantizar el logro de metas compartidas. El 
reto está en determinar las características de estos actores para encontrar el equilibrio entre las partes y asegurar la participación efectiva de todos, dotándoles de legitimidad y representatividad.

La transparencia en el proceso de gobernanza es otro elemento indispensable para estimular las relaciones entre las partes interesadas, al informar sobre las modalidades de participación, las normas, las reglas, las actividades y las metas compartidas. Al respecto, la participación puede abarcar desde el diagnóstico de la situación y el establecimiento de los objetivos del destino, considerando la transparencia y la rendición de cuentas para asegurar la confianza (Queiroz y Rastrollo-Horrillo, 2015). Igualmente, la gobernanza hace referencia a los medios y las acciones que van a permitir dirigir y controlar cuestiones que tengan relación con la gestión para la realización de objetivos; ya sean individuales u organizacionales, esto con la intención de crear bienes y valores públicos (De la Mora, 2015).

Ahora bien, el estudio de la gobernanza en ANP puede concentrar su interés en los diversos aspectos de las relaciones entre los actores gubernamentales y sociales (Martínez et al., 2015). De acuerdo con Valverde (2015), el estudio de la gobernanza en áreas protegidas ha cubierto varios temas estratégicos para la conservación y el aprovechamiento de los recursos, desde las políticas a la práctica, definiendo los financiamientos y los impactos de su regulación, por lo que se relaciona con el logro de los objetivos de la gestión y la efectividad en el manejo de dichos territorios.

En la literatura, el turismo en las áreas protegidas a menudo se concibe como un mecanismo que ayuda a aumentar el bienestar local y reducir la pobreza (Yergeau, 2019); sin embargo, su expansión y diversificación han tenido un impacto ambiental creciente en los ecosistemas protegidos (Canteiro et al., 2018). La sostenibilidad de un destino depende de la capacidad de las partes interesadas -gobierno, empresas y comunidades locales- para trabajar juntos en la aplicación de instrumentos de medición y regulación, que garanticen resultados favorables para la comunidad y el medio ambiente, de acuerdo con la Organización para la Cooperación y el Desarrollo Económicos (OCDE, 2010). En este sentido, es crucial promover una forma sostenible de turismo, que no sólo beneficie a la economía regional, sino que también ayude a proporcionar recursos y una base para el crecimiento económico de las comunidades locales (Pham-Do y Thanh, 2019). Así, las áreas naturales, cuando están protegidas, conservan el entorno natural y funcionan como espacios sociales en los que el turismo aporta mayores ingresos, empleo y apoyo financiero para la conservación (Oviedo-García et al., 2019).

Por lo anterior, De la Mora (2015) advierte que la realización de los procesos de gobernanza tiene que integrar de manera efectiva a la sociedad 
en general, al igual que a los diversos actores regionales; siendo considerable el aporte que se haga respecto al cuidado y la conservación de las áreas protegidas para lograr el mantenimiento de diversos servicios ambientales, así como integrar a actores que no participan de manera recurrente en el tema de la conservación. Consecuentemente, Valverde (2015) establece que indicadores como participación, equidad, responsabilidad y eficiencia podrán ayudar a determinar un buen nivel de gobernanza; en especial enfocado a la cuestión ambiental (donde se busca una relación conjunta con los principales actores internos y externos para el cuidado y la conservación del medio ambiente en los territorios).

Por otro lado, es importante dejar claro que en los territorios que presentan características de ANP resulta necesaria la determinación de las posibilidades de desarrollo turístico, en función de las condicionantes que presenten. En este sentido, la actividad turística debe contemplarse como una alternativa a la diversificación socioeconómica; de tal suerte que la adaptación a ésta representa la pauta para considerar la integración de los elementos que propician la gobernanza en dichos territorios, así como la correcta integración de la comunidad y el sector empresarial (Garzón y Ramírez, 2018). Además, aunque es innegable la importancia de la conservación de los territorios, también es sustancial establecer estrategias para que puedan aprovecharse sinergias por parte de los participantes de las comunidades y los agentes externos como gobierno, instituciones académicas, de investigación e inclusive comunidades aledañas a la misma, quienes participan igualmente en la conservación de las regiones.

Los beneficios que pueden generarse gracias a la correcta integración de comunidades alrededor de las actividades turísticas, como en el caso del turismo de naturaleza o ecoturismo, pueden ser económicos, ambientales y socio-culturales. De tipo económico se tiene la mejora y la diversificación de los servicios turísticos y el fomento para el desarrollo de productos locales, al igual que el reequilibrio de la población local al reducir las migraciones. En el ámbito ambiental, la buena integración comunitaria propicia el mantenimiento, la conservación y mejora de los espacios naturales; al igual que la concientización local sobre el patrimonio y la sensibilización ambiental hacia los turistas. Por último, en lo referente a la cuestión socio-cultural, se tiene la recuperación, revitalización y consolidación de la identidad y cultura local, el intercambio cultural y preservación de costumbres y tradiciones (Sánchez y Cebrián, 2015). 


\section{Resultados de la investigación}

Con relación a la tabla 1 , donde se muestran los aportes con más relevancia en torno a la asociatividad empresarial, que se realizó con el fin de poder analizar de manera más práctica y eficiente los elementos que integran dichos conceptos, se decidió tomar los principales componentes relacionados con el proceso de asociatividad empresarial para poderlos adaptar en un territorio que cuente con declaratoria de ANP, teniendo como resultado la tabla 2.

Tabla 2

Componentes del proceso asociativo aplicado en territorios

\begin{tabular}{ll}
\hline \multicolumn{1}{c}{ Autor } & Componente \\
\hline Rosales (1997) & Voluntad \\
\hline Poliak 2001, en Liendo y Martínez (2001) & Esfuerzo colectivo \\
\hline Liendo y Martínez (2001) & Creación de valor \\
\hline Tański et al. (2011) & Gobernanza \\
\hline Estrada (2016) & Territorio \\
\hline Posada et al. (2017) & Articulación \\
\hline Melo et al. (2017) & Conservación \\
& Productividad \\
& Sustentabilidad \\
\hline
\end{tabular}

Fuente: elaboración propia con base en (Posada et al., 2017), (Melo et al., 2017), (Estrada, 2016), (Liendo y Martínez, 2001), (Poliak 2001, en Liendo y Martínez, 2001) y (Rosales, 1997).

De acuerdo con la tabla 2, además de la gobernanza, los principales componentes que podrían incidir de manera particular y específica en la implementación de modelos asociativos en territorios declarados como ANP son la voluntad, el esfuerzo colectivo, la creación de valor, el territorio, la articulación, la productividad, la conservación y la sustentabilidad. Estos dos últimos componentes resultan de consideración prioritaria en el caso de ANP.

Igualmente, como producto de la revisión de los aportes y trabajos que se han realizado respecto a la asociatividad empresarial, se pudo efectuar un análisis más profundo no sólo de las partes que pueden integrar un modelo asociativo, sino también de los actores que de manera general intervienen y las principales consecuencias de dichos modelos. De manera sintetizada, se puede considerar la generación de un esquema 
conceptual de la asociatividad empresarial, así como las principales partes que lo conforman, los intermediarios y los resultados que se pueden obtener derivados de la conjugación de las partes antes mencionadas.

En la figura 1 se muestra el esquema conceptual del proceso de asociatividad: principales componentes, actores y resultados implementados y generados de dicho proceso. En primer lugar, se exponen los conceptos que conforman las características requeridas por territorios o comunidades para poder establecer un nivel mínimo de asociatividad. Dentro de

\section{Figura 1 \\ Proceso de asociatividad}

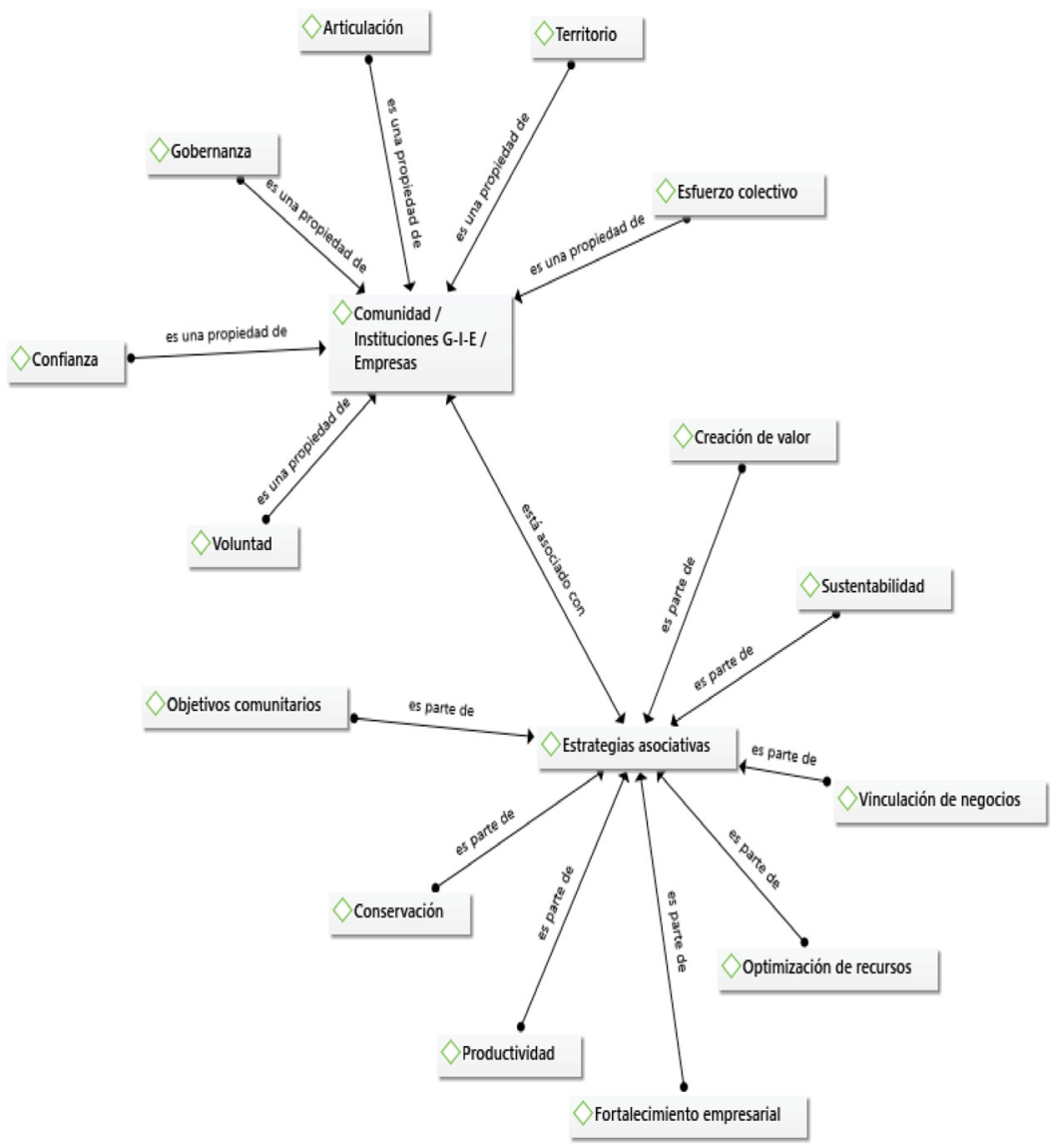

Fuente: elaboración propia con base en (Posada et al., 2017), (Melo et al., 2017), (Estrada, 2016), (Guerrero y Villamar, 2016), (Fajardo et al., 2016), (Cogollo y Arrieta, 2014), (Dini et al., 2007), (Liendo y Martínez, 2001), (Sectur, 2001), (Poliak 2001, en Liendo y Martínez, 2001), (Rosales, 1997). 
los componentes resaltan la determinación de un territorio, el grado de voluntad, la confianza, el esfuerzo colectivo, la gobernanza y la capacidad de articulación. Estos componentes deben ser desarrollados por tres actores principales, conformados por la comunidad, las instituciones (gubernamentales, educativas y de investigación) y el sector empresarial, donde este último funge como activador económico de los territorios.

En su conjunto, los actores implementarán estrategias asociativas para la detonación de múltiples beneficios como la optimización de recursos, el fortalecimiento empresarial, la vinculación de negocios, la productividad, la creación de valor y el cumplimiento de objetivos a nivel comunitario. Empero, reconociendo que la comunidad o territorio en el que se busca accionar un modelo asociativo puede presentar limitantes para el desarrollo de actividades económicas, como en el caso de las ANP, es necesario establecer estrategias focalizadas para la conservación y la sustentabilidad. En este sentido, el proceso es constante, no se termina, por lo que pueden instituirse estrategias para buscar un resultado en específico, más de uno o su mayoría.

En cuanto a la gobernanza, se pudo determinar que su activación en territorios requiere de otros elementos que son necesarios para su correcta evaluación; sobre todo cuando se trata de ANP, las cuales requieren de la participación integral y articulada de las partes para cumplir objetivos, tanto sociales como ecológicos, donde las autoridades cuentan con un papel determinante para su cumplimiento. No obstante, dichas autoridades siguen necesitando la participación de los integrantes de la comunidad para poder llegar a ese fin generalizado que, en definitiva, beneficie a los diversos actores en el territorio.

En la tabla 3 se muestran los principales elementos que hacen posible la detección de distintos niveles de gobernanza. Cuando ésta es favorable para el desarrollo de actividades, la participación en conjunto de actores territoriales y extraterritoriales hará que se potencialicen los resultados. De igual manera, en la tabla 4 se mencionan los elementos que se agregan a los antes mencionados con el fin de practicar una gobernanza efectiva en ANP buscando, al mismo tiempo, el cumplimiento de objetivos más particulares.

Como se muestra en la tabla 3, los principales componentes que ayudan a la correcta implementación de prácticas que fomentan la gobernanza en territorios van relacionados a la interacción entre los actores, no sólo de la comunidad, sino de actores ajenos a la misma, cuya intervención es parteaguas para el cumplimiento de los objetivos que se establezcan de manera conjunta.

Finalmente, en la tabla 4 se exponen los componentes que, sumados a los de la tabla 3, conformarán las sinergias pertinentes para que se generen 
Tabla 3

Principales componentes de la gobernanza

\begin{tabular}{lc}
\hline \multicolumn{1}{c}{ Autor } & Componente \\
\hline Arroyo y Palafox (2017) & Equilibrio de estado y sociedad \\
& Cooperación de actores \\
& Coherencia de acciones emprendidas \\
& Dinámica de trabajo entre personas \\
\hline Simancas y Ledesma (2017) & Redes de interacción \\
\hline
\end{tabular}

Fuente: elaboración propia con base en Arroyo y Palafox (2017) y Simancas y Ledesma (2017).

Tabla 4

Principales componentes de gobernanza para Áreas Naturales Protegidas

\begin{tabular}{lc}
\hline \multicolumn{1}{c}{ Autor } & \multicolumn{1}{c}{ Componente } \\
\hline $\begin{array}{l}\text { Abrams et al. (2003), citado en Martínez } \\
\text { et al. (2015) }\end{array}$ & Fortalecimiento de comunidades \\
\hline
\end{tabular}

Simancas y Ledesma (2017)

Toma de decisiones conjunta

Transparencia

De la Mora (2015)

Confianza

Fuente: elaboración propia con base en Abrams et al. (2003) citado en Martínez et al. (2015), Simancas y Ledesma (2017) y De la Mora (2015).

condiciones favorables sobre el cumplimiento de los objetivos comunitarios, establecidos en territorios que presentan la condicionante de ANP; considerando de manera especial los actores extraterritoriales que los mismos territorios y comunidades con dichas características demandan.

\section{Conclusiones}

Para fines de este trabajo se analizaron los constructos de asociatividad y gobernanza de manera individual, lo que permitió identificar los principales componentes de relación entre los mismos, donde la asociatividad presenta elementos necesarios para su ejecución como la sinergia a nivel comunidad, agentes extraterritoriales y el cumplimiento de objetivos en común; al igual que la gobernanza, donde esta última considera los agentes extraterritoriales como parte fundamental de dichos procesos. 
Una vez realizado el análisis correspondiente al concepto de asociatividad, se puede tener una perspectiva más clara de cuáles son los elementos que la conforman y qué es lo que antecede a su implementación. Primeramente, una de las cuestiones más destacadas que se tiene con los aportes que se analizaron es la consideración de dichos modelos como herramientas impulsoras del desarrollo económico de las localidades en las que se practican. En su diseńo, los modelos asociativos deben ofrecer una independencia jurídica y autonomía gerencial dentro del conjunto de empresas que la conformarán, siempre teniendo en cuenta el desarrollo de concordancias para su correcto funcionamiento, en especial a nivel comunitario, donde se podría tener un impacto más significativo.

Asimismo, se requiere que las partes que integran los modelos asociativos presenten un cierto nivel de compromiso, confianza e interés común para el beneficio en conjunto de la comunidad y el desarrollo propio. Al respecto, se debe considerar el impacto al desarrollo comunitario, siendo prioritaria una mejora en el nivel de vida de la población en el largo plazo. En este sentido, y en el caso particular de los servicios turísticos, es primordial tener en cuenta que los actores deben estar dispuestos y abiertos para el desarrollo de competencias propias que ayudarán a un mejor posicionamiento y participación en el mercado.

De manera práctica, se debe considerar que en la implementación de modelos de asociatividad, el uso de múltiples estrategias es imprescindible, partiendo de aquellas que tienen como referente a la gobernanza. De acuerdo con lo expuesto en este trabajo, la gobernanza es de suma importancia para el cumplimiento de los objetivos que se tengan de manera conjunta entre los diferentes actores del territorio. Por ende, entre los elementos más relevantes para la determinación de la gobernanza en los territorios se tiene la cooperación de actores, la coherencia en acciones emprendidas, la dinámica de trabajo que se pueda desarrollar entre la comunidad, la forma en cómo se dan las redes de interacción y, por supuesto, un alto manejo de confianza y compromiso.

Particularmente, tratándose de la gobernanza en territorios que presentan las condiciones de ANP, además de los elementos ya mencionados, es importante trabajar en la participación, la equidad y el desarrollo de la comunidad, basados en el logro de objetivos de gestión y efectividad en el manejo de estas áreas. Sin duda, la colaboración del entorno público y privado ayudará a la mejor integración de los actores y sinergias generadas en el entorno. Para tal caso, es crucial la integración de cada uno de los actores donde se respeten los valores que harán posible el cumplimiento de los objetivos deseados.

Por último, es importante considerar que se presentaron algunas limitantes para el desarrollo de un análisis más completo. En su mayoría, 
el material en el que se basó esta revisión habla de los procesos asociativos en industrias de gran impacto, como la cafetalera, textil, tabacalera, entre otras. Algunas de las investigaciones que sirvieron como base para la formación de este artículo implementan modelos de asociatividad empresarial, en los cuales sus actores claves ponen en marcha mecanismos de cooperación con otras regiones, no así en la misma comunidad en la que radican los participantes y el resto de los actores. En cuanto al estudio de la gobernanza, se analizaron artículos en los que se presenta el análisis del mismo en relación con las ANP; sin embargo, se advierte la carencia de estudios en México relacionados con la asociatividad empresarial en ANP, por consiguiente, se propone como futuras líneas de investigación para reforzamiento y complemento de lo expuesto en el presente artículo.

\section{Fuentes consultadas}

Arguello Núñez, León; Rendón Guerra, Gina y Pérez, Mario Alejandro (2017), "El proceso asociativo como ventaja competitiva en las microempresas, caso Quevedo-Ecuador", ROCA. Revista Científico-educacional de la provincia Granma, 13 (3), Bayamo, Universidad de Granma, pp. 1-11.

Arroyo Delgado, María y Palafox Muñoz, Alejandro (2017), “Turismo, gobernanza y sustentabilidad social en el Área Natural Protegida de Cabo Pulmo, B.C.S.", en Rubén Ibarra Reyes; Eramis Bueno Sánchez; Rubén Ibarra Escobedo y José Luis Hernández Suárez, La humanidad frente a los desafios del capitalismo decadente, Zacatecas, Universidad Autónoma de Zacatecas, pp. 112-127.

ATLAS.ti (2017), “ATLAS.ti Qualitative Data Analysis”, version 8, Berlin, ATLAS.ti Scientific Software Development GmbH.

Bada Carbajal, Lila Margarita; Rivas Tovar, Luis Arturo y Littlewood Zimmerman, Herman Frank (2017), "Modelo de asociatividad en la cadena productiva en las Mipymes agroindustriales", Contaduria y Administración, 62 (4), Ciudad de México, Universidad Nacional Autónoma de México, pp. 1100-1117.

Barba Sánchez, Virginia y Molina Ramírez, Ericka (2008), “Desarrollo regional por medio de un cluster ecoturístico en la Huasteca Norte Potosina", Revista da Micro e Pequena Empresa, Campo Limpo 
Paulista, 2 (1), Campo Limpo Paulista, Faculdade Campo Limpo Paulista-FACCAMP, pp. 13-27.

Canteiro, Marcelo; Córdova-Tapia, Fernando y Brazeiro, Alejandro (2018), "Tourism impact assessment: A tool to evaluate the environmental impacts of touristics activities in Natural Protected Areas", Tourism Management Perspectives, vol. 28, Queensland, Griffith University, pp. 220-227, doi: https://doi.org/10.1016/j. tmp.2018.09.007

Cogollo, Kenia Victoria y Arrieta Flores, Rosaura (2014), “La organización empresarial asociativa como estrategia para la inclusión productiva de las pequeñas y medianas empresas", Palobra. Palabra que Obra, núm. 14, Cartagena, Universidad de Cartagena, pp. 100-117.

De la Mora de la Mora, Gabriela (2015), "Redes sociales y Áreas Naturales Protegidas en la Zona Metropolitana de Monterrey, Nuevo León", Economía, Sociedady Territorio, 15 (49), Zinacantepec, El Colegio Mexiquense, A. C., pp. 747-778.

Dini, Marco (2010), "Competitividad, redes de empresas y cooperación empresarial”, documento de la Serie Gestión Pública núm. 72, Santiago de Chile, Naciones Unidas-CEPAL.

Dini, Marco; Ferraro, Carlo Alberto y Gasaly, Carolina (2007), "Pymes y articulación productiva. Resultados y lecciones a partir de experiencias en América Latina", documento de la Serie Desarrollo Productivo núm. 180, Santiago de Chile, Naciones Unidas-CEPAL.

Estrada Arbeláez, Laura (2016), "Asociatividad para el desarrollo de los territorios rurales: Una mirada más allá de la competitividad", en Andrés Molano Rojas, Colombia: Encrucijadas y Perspectivas. Una agenda para el avance institucional y el desarrollo, Bogotá, CEPBanco de la República-Biblioteca Luis Ángel Arango, pp. 129-196.

Fajardo Vaca, Ligia; Fernández Ronquillo, Mario; Vásquez Fajardo, Carlos, Toscano Ruiz, Darwin y Fajardo Vaca, Luis (2016), "La asociatividad micro empresarial, como mecanismo productivo sostenible: caso Cantón Milagro-Ecuador", Universidad, Ciencia y Tecnología, 20 (78), Caracas, Universidad Nacional Experimental Politécnica Antonio José de Sucre (UNEXPO), pp. 4-13. 
Fundeanu, Daniela y Sandu Badele, Cosmin (2014), "The impact of regional innovative clusters on competitiveness", Procedia. Social and Behavioral Sciences, vol. 124, Ámsterdam, Education Resources Information Center, pp. 405-414.

Gallicchio, Enrique (2004), "El desarrollo local: ¿`cómo combinar gobernabilidad, desarrollo económico y capital social en el territorio?", Cuadernos del CLAEH, 27 (89), Montevideo, Centro Latinoamericano de Economía Humana, pp. 55-68.

Galvez-Acevedo, Omar; Martínez-Castañon, José; Cano-Lazarte, Mercedes y Raymundo-Ibañez, Carlos (2020), "An associative ecotourism model aimed at optimizing resources for tourism chain smes in the highland communities of the Peruvian Andes", International Journal of Social Science and Humanity, 10 (3), Singapure, IACSIT Press, pp. 82-86. DOI: 10.18178/ijssh.2020.10.3.1019

Garzón García, Rafael y Ramírez López, María Luisa (2018), "Las áreas protegidas como territorios turísticos: análisis crítico a partir del caso de los parques naturales de la sierra morena andaluza", Cuadernos de Turismo, núm. 41, Murcia, Universidad de Murcia, pp. 249-277.

Guerrero Bejarano, María Auxiliadora y Villamar Cobeña, Josué Andrés (2016), "La importancia de la asociatividad para el desarrollo", INNOVA Research Journal, 1 (11), Quito, Universidad Internacional del Ecuador, pp. 105-119.

Gutiérrez Mejía, Luis Alberto y Silva Aristizabal, María Isabel (2013), "La asociatividad territorial, estrategia para el desarrollo empresarial y regional de Colombia”, Medellín, Universidad de Antioquia, pp. 1-14, <http://www.redpilares.net/sobre-la-red/ Documents/MEJ\%C3\%8DA_ARISTIZABAL_LA\%20ASOCIATIVIDAD\%20TERRITORIAL\%20ESTRATEGIA\%20 PARA\% 20EL\%20DESARROLLO\% 20\%20EMPRESARIAL\%20Y\%20REGIONAL\%20DE\%20COLOMBIA.pdf>, 24 de julio de 2020 .

Hanafiah, Mohd Hafiz; Jamaluddin, Mohd Raziff y Zulkifly, Muhammad Izzat (2013), "Local community attitude and support towards tourism development in Tioman Island, Malaysia", Procedia-Social and Behavioral Sciences, vol. 105, Ámsterdam, 
Education Resources Information Center, pp. 792-800, doi: https://doi.org/10.1016/j.sbspro.2013.11.082

Ibañez Pérez, Reyna (2014), "Turismo y sustentabilidad en pequeñas localidades costeras de Baja California sur (BCS)”, El Periplo Sustentable, núm. 26, Toluca, Universidad Autónoma del Estado de México, pp. 67-101.

Liendo, Mónica y Martínez, Adriana (2001), “Asociatividad. Una alternativa para el desarrollo y crecimiento de las Pymes", ponencia presentada en las sextas jornadas "Investigaciones en la Facultad" de Ciencias Económicas y Estadística, 9 y 10 de noviembre, Rosario, Argentina.

Martínez, Nain; Brenner, Ludger y Espejel, Ileana (2015), "Red de participación institucional en las Áreas Naturales Protegidas de la península de Baja California”, Región y sociedad, 27 (62), Hermosillo, El Colegio de Sonora, pp. 27-62.

Melo Torres, Ligia Inés; Melo Torres, María Mercedes y Fonseca Pinto, Dora Esther (2017), "The Associativity: a local development strategy for Ocamonte (APCO) coffee growers in Santander, Colombia”, Acta Agronómica, 66 (4), Bogotá, Universidad Nacional de Colombia, pp. 538-543.

Muñoz Hernandez, Luis David (2019), "Asociatividad empresarial como estrategia para la competitividad de las Pymes del sector moda de México y Colombia”, tesis de maestría, Universidad Libre de Pereira, Facultad de Ciencias Económicas, Administrativas y Contables, Programa de Contaduría Pública, Pereira, <https:// repository.unilibre.edu.co/bitstream/handle/10901/17822/ ASOCIATIVIDAD\%20EMPRESARIAL \%20COMO\%20 ESTRATEGIA\% 20 PARA \% 20 LA.pdf? sequence $=1 \&$ isAllowed=y>, 24 de julio de 2020.

Murphy, Peter (1983), "Tourism as a community industry-an ecological model of tourism development", Tourism Management, 14 (3), Ámsterdam, Elsevier, pp. 180-193, doi: https://doi.org/10.10 16/0261-5177(83)90062-6

OCDE (Organización para la Cooperación y el Desarrollo Económicos) (2010), "Tourism trends and policies", París, OECD Publishing, doi: https://doi.org/10.1787/6b47b985-en 
OMT (Organización Mundial del Turismo) (1999), “Código Ético Mundial para el Turismo”, Madrid, OMT/Naciones Unidas, <https:// webunwto.s3.eu-west-1.amazonaws.com/s3fs-public/2019-10/ gcetpassportglobalcodees.pdf>, 24 de julio de 2020.

Oviedo-García, M. Ángeles; Vega-Vázquez, Manuela; Castellanos-Verdugo, Mario y Orgaz-Agüerac, Francisco (2019), "Tourism in protected areas and the impact of servicescape on tourist satisfaction, key in sustainability", Journal of Destination Marketing \& Management, vol. 12, Londres, Elsevier Ltd, pp. 74-83, doi: https://doi.org/10.1016/j.jdmm.2019.02.005

Palomino Villavicencio, Bertha; Gasca Zamora, José y López Pardo, Gustavo (2016), "El turismo comunitario en la Sierra Norte de Oaxaca: pespectiva desde las instituciones y la gobernanza en territorios indígenas", El Periplo Sustentable, núm. 30, Toluca, Universidad Autónoma del Estado de México, pp. 6-37.

Pham-Do, Kim Hang y Thanh, Thuy Thi (2019), “Tourism in marine protected areas: A view from Nha Trang Bay, Vietnam", Tourism Management Perspectives, vol. 33, Londres, Elsevier, pp. 1-9, doi: https://doi.org/10.1016/j.tmp.2019.100623

Posada Arrubia, Adriana; Campuzano, Antonio Esteban y Berrocal Mendoza, Ana Elena (2017), "Gestión territorial mediante estrategia de asociatividad para Casanare-Colombia", Revista U.D.C.A Actualidad \& Divulgación Científica, 20 (2), Bogotá, Ediciones U.D.C.A., pp. 445-456.

Queiroz, Françoise y Rastrollo-Horrillo, Ángeles (2015), “El estado del arte en gobernanza de destinos turísticos", Tourism \& Management Studies, 11 (2), Faro, Universidade do Algarve, pp. 47-55.

Rosales, Ramón (1997), "La asociatividad como estrategia de fortalecimiento de las PYMES”, Revista SELA, núm 51, Caracas, Sistema Económico Latinoamericano y del Caribe, pp. 1-12.

Sánchez Jasso, Jessica Mariana y Cebrián Abellán, Francisco (2015), "Turismo de naturaleza en áreas protegidas de México; una propuesta de conservación, aprovechamiento y desarrollo local en El Nevado de Toluca", Cuadernos de Turismo, núm. 36, Murcia, Universidad de Murcia, pp. 339-365. 
Sánchez Serrano, Rolando (2010), "El capital social y sus efectos socieconómicos y políticos", Tinkasos, 13 (supl. 1), La Paz, Fundación del Programa de Investigación Estratégica en Bolivia, pp. 191-199, <http://www.scielo.org.bo/scielo.php?script=sci_arttext\&pi $\mathrm{d}=$ S1990-74512010000300009>, 24 de julio de 2020.

Sectur (Secretaría de Turismo) (2001), "Programa de competitividad, Integración de Agrupamientos Turísticos Competitivos 'Clusters Turísticos", Serie de documentos técnicos, fascículo 5, Ciudad de México, Sectur.

Simancas Cruz, Moisés y Ledesma González, Oswaldo (2017), “El papel de los Stakeholders en los procesos de gobernanza y renovación de las áreas turisticas de litoral", Estudios y Perspectivas en Turismo, 26 (2), Buenos Aires, Centro de Investigaciones y Estudios Turísticos (CIET), pp. 349-369.

Tañski, Nilda; Clérici, Carmen y Báez, Lucio (2011), "La gobernanza en la gestión asociativa entre PYMES: Sector de la foresto-industria y actividades relacionadas. Provincia de Misiones, Argentina”, Dimens. empres., 9 (1), Barranquilla, Universidad Autónoma del Caribe, pp. 102-114.

Valverde Garnica, Álvaro (2015), “Gobernanza ambiental en áreas naturales protegidas. El caso del Parque Nacional y Área Natural de Manejo Integrado Cotapata", Integra Educativa, 8 (3), La Paz, Instituto Internacional de Integración, pp. 71-85.

Varisco, Cristina (2008), "Innovación y asociatividad en la actividad turística. Las empresas de servicios en Pinamar", ponencia presentada en IX Jornadas Nacionales y III Simposio Internacional de Investigación-Acción en Turismo, 22-24 mayo, San Juan, Argentina.

Vázquez-Barquero, Antonio (2005), "Las nuevas fuerzas del desarrollo", Barcelona, Antoni Bosch.

Yergeau, Maria-Eeve (2019), "Tourism and local welfare: A multilevel analysis in Nepal's protected areas", World Development, vol. 127, Londres, Elsevier, doi: https://doi.org/10.1016/j. worlddev.2019.104744 
Recibido: 30 de enero de 2020. Reenviado: 14 de julio de 2020. Aceptado: 25 de agosto de 2020.

Dulce María Lucero López. Contador Público por el Instituto Tecnológico de La Paz con especialidad en finanzas empresariales. Actualmente cursa el último semestre de la Maestría en Administración Estratégica en la Universidad Autónoma de Baja California Sur, México, con especialidad en Políticas Públicas y Desarrollo Regional.

Alberto Francisco Torres García. Doctor en Relaciones Transpacíficas por la Universidad de Colima. Actualmente es profesor-investigador de tiempo completo en el Departamento Académico de Economía de la Universidad Autónoma de Baja California Sur. Es miembro del Sistema Nacional de Investigadores nivel I y cuenta con Perfil PRODEP. Es miembro del Cuerpo Académico de Políticas Públicas y Desarrollo Económico. Sus áreas de investigación se relacionan con el estudio de la competitividad y los negocios internacionales. Entre sus más recientes publicaciones destacan, como autor: "El fortalecimiento de la facilitación comercial en México a partir del Tratado Integral y Progresista de Asociación Transpacífico", México y la cuenca del pacífico, 7 (21), Guadalajara, Universidad de Guadalajara, Centro Universitario de Ciencias Sociales y Humanidades, Departamento de Estudios del Pacífico, pp. 145-176 (2018); como coordinador: Turismo, desarrollo económico y sustentabilidad en Baja California Sur, Sinaloa, Universidad Autónoma de Sinaloa (2018); y como coautor: "Análisis de oportunidades para el desarrollo de actividades de turismo alternativo en la región norte de Baja California Sur (México)", 3C Empresa: investigación y pensamiento crítico, 7 (1), Alicante, Área de Innovación y Desarrollo S. L., pp. 32-48 (2018). 